\title{
THE NERVOUS CORRELATE OF ATTENTION.
}

\author{
BY PROFESSOR MAX MEYER,
}

University of Missouri.

Introduction.

ConTants.

I. The Fundamental Laws of Nervous Function.

II. The Uniqueness of Pain Reactions.

III. The Susceptibility of Connecting Neurous.

IV. Automatic Action.

V. Vividness and Intensity.

VI. Attention as a Faculty.

\section{INTRODUCTION.}

In a previous article in this Review ${ }^{1}$ I developed a theory of the relation between nervous function and consciousness. However, I had to be very brief in the treatment of some aspects of this relation. It was then my chief intention to combat that mythological theory of pleasantness-unpleasantness, according to which Pleasantness and Unpleasantness are two ghost-like entities sitting within our brain - armed, one with shears, the other with a soldering iron (according to a variant: with a stamping iron) - breaking and making connections at will. It is my conviction that, just as it is possible to comprehend the reciprocation of the action of a steam engine as being caused by the engine itself, so it is possible to comprehend the modification to which the instinctive action of a higher animal is subject, as being governed by the fundamental laws of the nervous system itself.

I shall now treat in more detail a number of questions concerning the relation of nervous function and consciousness which I could not take up previously without leading the reader's attention too far away from the problem of pleasantness-unpleasantness. But we shall no more restrict ourselves now to an exclusive discussion of the non-affective consciousness than we

1 ' The Nervous Correlate of Pleasantness and Unpleasantness,' PsychocogICAI, REVIEW, XV., 4, 5, 20I-216, 292-322, I908.

$35^{8}$ 
restricted ourselves then to an exclusive discussion of pleasantness-unpleasantness. It is, of course, only by abstraction that we divide our consciousness into classes to be kept in different compartments. Every application to actual life forces us to take into consideration the mutual relations of these classes, although our scientific interest may momentarily seem to be confined to one of them.

Meanwhile Titchener's Psychology of Feeling and Attention has been made public. If there is anyone whom the historical part of my previous article did not convince that the traditional methods of investigating feeling - no matter whether experimental or otherwise - have reached a cul-de-sac, Titchener's book will convince him. The author's conclusion is decidedly pessimistic, one might even say desperate. He confesses "to a feeling of unpleasantness, tension and depression. We know so very little of the subject of these Lectures, and the work that we have found to do will take so long in the doing!" This will hardly encourage any student to devote himself to an investigation of feeling. What we need is a new point of view. I offer my own view with the hope that it will give the reader as much relief, encouragement, and pleasantness as it has given me.

\section{The Fundamental Laws of Nervous Function.}

Let me begin with an abbreviated statement of the laws of nervous function as found on pages $300 \mathrm{ff}$. of my previous article.

I. Let us assume that any point where two or more neurons are connected, functions like a valve which permits fluid to pass through only in one, not in the opposite direction, and that this valve opens only in the direction towards motor points of the body.

2. Let us assume that any neuron system has functional properties analogous to those of a pipe system filled with a fluid, one end of the pipe system being usually closed (the sensory point), the other end being under the influence of negative pressure, or suction, tending to produce a current in the direction of this end, the motor point. 
3. Let us assume that ordinarily no movement can take place in the pipe system because the sensory end is closed; that the application of a weaker or stronger stimulus means the same as the opening to a lesser or greater extent of the closed end of such a pipe system, thus permitting fluid to escape through the motor point (or points).

4. Let us assume that the resistance suffered by the fluid in moving through the pipe system depends in the ordinary way on the length and the width of the pipe.

5. Let us assume that the pipe is widened by the current in proportion to its intensity and duration, and that, while unused, the pipe has a tendency to grow narrower; and that this susceptibility of the pipe is the more pronounced the 'higher' the connecting neuron.

To these assumptions was added on page 303 a sixth one, the most important one in so far as it serves to explain the function which is analogous to that of a steam-engine's valve-gear - a function for which thus far mythological explanations have been offered rather than scientific ones.

6. Nervous currents are subject to a law analogous to the dynamic law that hydraulic pressure is less than hydrostatic pressure, of which the jet pump is a familiar illustration.

For the reason already mentioned I did not attempt in my previous article to justify these assumptions except by applying them to habit formation and to the rôle played by pleasantness-unpleasantness in mental life. I shall now give additional reasons why it seems to me to be wise to make these assumptions rather than others.

A nervous process is generally regarded as a chemical process. But of what kind? It is most frequently compared with the burning of a fuse. In my opinion there is no analogy in this case at all. But before giving reasons why I regard the analogies offered by myself as scientifically valuable, let me say a word in reply to the possible question, why we should use analogies rather than wait until other sciences are able to tell us exactly what the nervous process is.

I take it for granted that a teacher of psychology wants to give his students more than a mere classification of mental 
states, that he wants to show them how the laws of mental life can be understood as a part of the natural laws governing a highly developed organic being. What does he answer to the student's question in what manner the nervous system is modified when instincts are modified into habits? Unless he can offer to the student a picture - however hypothetical — of the laws of nervous activity, he is obliged to offer mythological entities making and breaking connections at will. I am far from dogmatically asserting that it is inconceivable that pleasantness and unpleasantness make and break connections in the brain; but this is an entirely empty, purely formal statement, as long as we are not told the exact nervous or mental conditions under which pleasantness and unpleasantness themselves occur. We cannot explain to the student how our actions are determined by our mental life without a detailed picture of nervous activities. Since experimental physiology at present does not offer us such a picture, at least not one which serves the purposes of the psychologist, we have to develop one ourselves. We cannot wait for physiological discoveries which may never be made.

So much in excuse of my making assumptions. Let us return to the statement that we cannot picture a nervous process as the burning of a fuse. A fuse burns in either direction. A nervous process, it is true, if started in a neuron, is propagated in both directions within that neuron. But it does not seem probable, so far as I can judge the experimental evidence, that a nervous process can normally pass from one neuroa into another neuron which is nearer to a sensory point of the body. If this were otherwise, it would be possible for central excitations to produce normal sensory processes in our sense organs. This seems improbable, in spite of so-called synesthesias, which in my opinion must be otherwise explained. I have therefore accepted the first assumption that any point of connection functions like a valve which permits fluid to pass only in one, not in the opposite direction.

Another reason why it seems confusing to picture the nervous process as the burning of a fuse in this: If a fuse branches out, the burning would proceed along all the branches, regard- 
less of differences in the length of the branches. But the nervous process does not branch out in this manner. However important the law of nervous diffusion may be, this kind of diffusion - analogous to the burning of a branching fuse-does not exist. Another point of disagreement is the following: A fuse cannot be made to continue burning for a considerable time by mere persistence of the cause of the burning. But a nervous process can be made to continue by continued stimulation, although not for an indefinite time.

It seems to me most probable that the nervous process is of the same nature as the migration of ions in an electrolyte. The migration of ions is accompanied by electrical phenomena which permit a diagnosis of the migration and a measurement of the flux of ions. Similarly the nervous process is accompanied by electrical phenomena. Migration of ions can be caused by the electric current : so can the nervous process. But migration of ions is also dependent on and caused by many other physical and chemical conditions: so is the nervous process. In spite of these and other agreements, I did not in my second assumption picture the nervous process as a migration of ions for the following reason. Some one who might think that he knows that the nervous process is not a migration of ions, might forget that $I$ offered merely a picture and might argue against the whole theory. No one, surely, will forget that speaking of the nervous system as a pipe line is merely using a picture, an analogon, so that his refusal to admit that it actually is a pipe line, cannot be made an argument against the theory here offered.

A possible confusion of terms may be anticipated and prevented by explanation. When we think of a fluid, first at rest and under a constant tension ('negative pressure'), then moving, the word ' velocity' may be used in two different meanings, namely, as standing for the flux or for the velocity of the propagation of the relief of tension. In a pipe closed at one end, and with suction applied to the other end, the whole column of fluid is under tension. As soon as the closed end is opened, the tension is relieved at this end, and the flux may begin. But at any distant point the flux can begin only after the relief of tension 
has occurred at that point; and the whole column of fluid can be in motion only after the relief of tension has traveled all through the pipe and reached the open end. The velocity with which this relief of tension travels is analogous to the velocity of the propagation of the nervous process as usually measured and found to be about $60 \mathrm{~m}$. per second or less. It is independent of the size of the opening at the sensory point, that is, of the intensity of stimulation. On the other hand, the term velocity might be used in the sense of 'number of fluid units which pass a cross-section of the pipe in one second.' Velocity in the former sense does not concern us in our present discussion. In the latter sense it is an important concept; but owing to the equivocality of the term, I shall avoid it in the future and use the word flux. Flux, then, is analogous to intensity of the nervous process.

The assumption of a constant suction at the motor end of the pipe line is merely analogous to the fact that the nervous system, unless exhausted, is always ready to respond to stimulation. It does not, of course, imply that the muscle fibers act like an exhaust pump. I do not conceive either of the sensory or of the motor points of the nervous system as points where energy is transmitted, but as points where the performance of work is made possible by the fulfilment of a necessary condition. Sensory stimulation may be compared with the immersing of the zinc in a plunge-battery - the resulting migration of ions is of course not the equivalent of the mechanical energy of the motion of the zinc, although the greater the immersing mcvement of the zinc, the greater the flux of ions. Innervation of the muscle may be compared with a signal for action given to a body of men by means of an electric bell. Again there is no energy equivalent. This will explain the third assumption.

The fourth assumption needs no explanation. The fifth assumption shows clearly the advantage of using a mechanical picture as analogon of the nervous process. We can easily conceive a pipe line, the inner wall of which consists of a material that may be washed out by action of the fluid. Just recall a limestone cave. And it is also easy to think of a deposit on the walls resulting from lack of use. Let us call this property 
of the pipe, enabling its resistance to decrease or increase, the susceptibility of the pipe. This susceptibility I assumed to be the greater the higher the connecting neuron in question. I did this for the following reasons: ( $r$ ) Our sense organs do not seem to respond more promptly to stimuli to which they have often been exposed. Rather the opposite. Blind-born persons who for years have made little use of their eyes, have after operation no inferior, but rather a superior visual sensitivity. It seems best, then, to assume that the sensory (and also the motor) neurons have no susceptibility as above defined, but that this belongs only to the connecting neurons. (2) It is generally accepted that the higher nerve centers suffer more easily from pathological influences than the lower ones. I have therefore accepted the assumption that the higher connecting neurons have a greater normal susceptibility than the lower ones.

The sixth assumption shows again the advantage of using a mechanical picture of the nervous process. Everybody is familiar with the principle underlying the function of a jet pump. No vague conception, but a clear image of a familiar instrument guides our thought. What is possible in a mechanical system - the attraction of a weaker process by a stronger. one - must also be possible under physiological laws, which are far more complicated than those of mechanics. Of course, there is this difference between the mechanical instrument in our theory and the ordinary jet pump, that in the latter the moving force is pressure, in our case it is suction, which requires a somewhat different mechanical arrangement.

The second and sixth assumptions would not be possible without the first. If the nervous process could pass in either direction through any connecting point of neurons, flux might easily occur from one motor point in the direction of another, as soon as the force of suction should fail to be exactly alike at all motor points. And the change from hydrostatic to hydraulic pressure would also cause, by attraction, flux from motor points to a motor point. This seems to lead to impossible complications, and is an additional reason for making the first assumption. 


\section{The Uniqueness of Pain Reactions.}

Instinctive reactions upon pain show a remarkable difference from other instincts. Unless this difference is clearly understood, pain reactions present a serious difficulty to our theory of nervous function. We have assumed that a stronger nervous process attracts a weaker one. A strong and continuous pain process, for example one originating from a sore tooth, might then be expected to attract all other nervous processes from whatever sense organs they originate. Since the pain process is there first, the new nervous processes, it seems, can never attain constancy of flux, but as soon as they originate must join the pain process. The flux of the latter, therefore, is again and again increased at points other than sensory points of the body. Must we not conclude then from our theory that a person who has a strong toothache should uninterruptedly be engaged in the same muscular activity, and that a toothache should be a highly pleasant experience?

To some extent this difficulty can be met theoretically by the following explanation. Touching and pressing upon the tooth does probably weaken the sensation of pain somewhat. But as soon as our hand has now begun to respond to some other stimuli acting upon our eyes, ears, or other sense organs, the pain stimulus starts again with renewed vigor, and the' renewed process coming from the pain point deflects these other nervous processes soon after they have become constant, with resulting unpleasantness.

Of much more importance, however, is the following fact, which makes it plain why a strong sensation of pain must be unpleasant although the stimulation may continue strong. If the instinct of, say, swallowing is properly stimulated, the subject swallows. If the stimulus continues, the subject does not scratch himself behind the ear or spit, but swallows again and again. But the instinctive response to a pain stimulus is not a repetition of the first reaction. If $I$ perceive pain at a definite point of my body, I perhaps move first my finger toward that point. If in spite of this movement the pain should continue, could it be of much value to me to repeat the movement? This movement obviously failed to remove the cause of the sen- 
sation of pain. Another kind of movement must therefore occur, as a biological necessity, - and actually does occur, instinctively. If the pain still continues, a third kind of movement occurs; and so on. We may describe the difference between the instinctive pain reaction and other instincts thus: If the pain reaction is repeated because of a continuance of the stimulus, this does not mean that the same movement is repeated, but a different movement occurs each time. The nervous process, although starting from the same sensory point, does not, as in other instincts, continue to flow over the same path in the same motor direction, but suddenly takes a different path, after a while, if the stimulation continues, again a different path, and so on. Everybody knows what dancing and writhing movements result from continued pain, wherever the stimulus may be located. By what mechanism this recurrent change of action is brought about, I am unable to suggest. But the fact of its being brought about by some structural or functional peculiarity of our pain nerves cannot be doubted.

If we apply this to the above case of a sore tooth, we understand why a continued toothache cannot be pleasant. We at first thought of the nervous process resulting from the stimulation as a process going on continuously over the same path, and forcing all later arising and weaker processes to join it. We now see that the pain process is not one long-continued process, but many different processes succeeding each other. Every time another pain reaction occurs, the new nervous process interferes with the other processes going on at that moment, coming from various sense organs, by deflecting them. Unpleasantness must therefore be felt.

What has been said of pain reactions applies largely also to the instinctive reactions upon those other sense impressions which are harmful to the body, as bitterness or acidity. Having got a bitter substance into our mouth, we at first simply spit; but if the bitter sensation continues with any strength, we instinctively do many other things, wash our mouth with our tongue and with our fingers, put other things in our mouth, drink water, etc. For the same reason, therefore, an uninterrupted stimulus of bitter or sour is not likely to produce a sensation accompanied by pleasantness. 


\section{The Susceptibility of Connecting Neurons.}

That property of the connecting neurons which in our fifth assumption we called susceptibility, is of much significance in several respects. In my previous paper I did not make any statement concerning a definite ratio of the susceptibility of higher and lower neurons, but merely stated that the susceptibility of the higher neurons was greater. The assumption of definite values, however, leads to important consequences.

It is necessary that we distinguish between the two kinds of susceptibility by giving them definite names. Let us call positive susceptibility that property of a neuron which consists in a decrease of resistance in consequence of carrying a current, negative susceptibility that property which consists in an increase of resistance in consequence of lack of function.

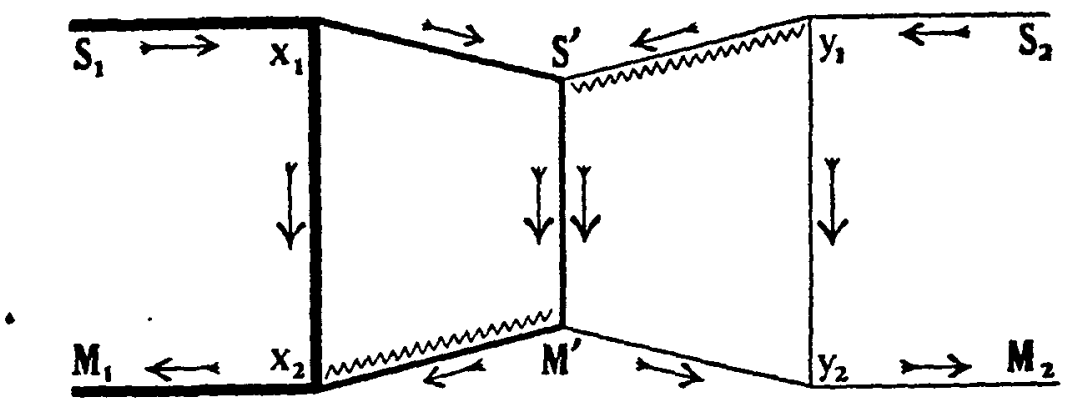

FIG. x.

Let us arbitrarily assume that the positive susceptibility of each of the three neurons $x_{1} S^{\prime}, S^{\prime} M^{\prime}$ and $M^{\prime} x_{2}$ in Fig. $I$ is three times that of the neuron $x_{1} x_{2}$; and further, that at the earliest time when all these neurons have reached their complete development, the resistance of $x_{1} x_{2}$ is equal to that of each of the neurons $x_{1} x^{\prime}, S^{\prime} M^{\prime}$ and $M^{\prime} x_{2}$. Suppose now that the point $S^{\prime}$ is stimulated. The flux is distributed according to the resistances offered by the paths. The resistance of the path $x_{1} S^{\prime} M^{\prime} x_{2}$ is three times the resistance of the path $x_{1} x_{2}$. The flux at any point of the path $x_{1} x_{2}$ must therefore be three times the flux at any point of the path $x_{1} S^{\prime} M^{\prime} x_{2}$. The resistance of both paths must decrease because of the current in both. If the decrease 
depended on the flux alone, it would be three times as great in $x_{1} x_{2}$ as in $x_{1} S^{\prime} M^{\prime} x_{2}$. But we assumed the positive susceptibility of the latter path to be three times that of the former. The decrease of the resistance is therefore exactly the same at any point of $x_{1} S^{\prime} M^{\prime} x_{2}$ as at any point of $x_{1} x_{2}$. Consequently, when the stimulation at $S_{1}$ ceases, although the resistance of all the four neurons in question has decreased, we find again that the resistance of $x_{1} x_{2}$ is equal to the resistance of each of the neurons $x_{1} S^{\prime}$, $S^{\prime} M^{\prime}$ and $M^{\prime} x_{2}$. In this consideration, for simplicity's sake the existence of the path $M^{\prime} y_{2}$ has been neglected. The flux from $S^{\prime}$ to $M^{\prime}$ must of course divide at the point $M^{\prime}$. But most of it must take its way toward $x_{2}$, much less towards $y_{2}$, because of the attraction by the current going on in $x_{1} x_{2} M_{1}$.

The negative susceptibility has not yet become effective. Now, how will the relative resistance of the four neurons $x_{1} x_{2}$, $x_{1} S^{\prime}, S^{\prime} M^{\prime}$ and $M^{\prime} x_{2}$ be found after some time? If the negative susceptibility does not differ in these neurons, no change will occur. If it is greater in each of the three neurons $x_{1} S^{\prime}$, $S^{\prime} M^{\prime}$ and $M^{\prime} x_{2}$ than in $x_{1} x_{2}$, the resistance of the path $x_{1} S^{\prime} M^{\prime} x_{2}$ would soon become greater than that of $x_{1} x_{2}$. Is this probable? To some extent it seems to be true, for instincts cannot be trained, that is, modified, so well at a time much later than their natural appearance. "Was Hänschen nicht lernt, lernt Hans nimmermehr!" But special experiment alone can give an answer to the question as to just how much greater the negative susceptibility of higher neurons is than that of lower ones.

With respect to the positive susceptibility, if we had not made the assumption that in each of the neurons $x_{1} S^{\prime}, S^{\prime} M^{\prime}$ and $M^{\prime} x_{2}$, it was three times that of $x_{1} x_{2}$, but the assumption that it was less, say only twice as great, what would have been the consequence? Obviously this: At the time when the stimulation at $S_{1}$ ceased, the resistance of the neuron $x_{1} x_{2}$ would have been found - not equal to, but only two thirds of the resistance of each of the neurons $x_{1} S^{\prime}, S^{\prime} M^{\prime}$ and $M^{\prime} x_{2}$. Repeated stimulation would then result in a pronounced widening of the channel $x_{1} x_{2}$ and a relative or absolute (because of the negative susceptibility) narrowing of the channel $x_{1} S^{\prime} M^{\prime} x_{2}$. This is the case spoken of very briefly on page $3^{02}$ of my previous 
paper as a habit which is merely a strengthened instinctstrengthened in such a way that any later modification becomes impossible.

If the ratios of the positive and of the negative susceptibility are assumed to be exceedingly different, a habit can be conceived which is merely a strengthened instinct, but which can easily be modified at any time. Let us assume that the positive susceptibility of each of the neurons $x_{1} S^{\prime}, S^{\prime} M^{\prime}$ and $M^{\prime} x_{2}$ is ten times that of $x_{1} x_{2}$; and that the negative susceptibility of the former is but a trifle larger than that of the latter. Now suppose that the point $S_{1}$ is very frequently stimulated, each time for a considerable length of time, and with but short pauses between the stimulations. It is clear that then - in spite of the flux being at first at any point of $x_{1} x_{2}$ three times as great as the flux at any point of the path $x_{1} S^{\prime} M^{\prime} x_{2}$ - the resistance of the path $x_{1} S^{\prime} M^{\prime} x_{2}$ will finally be only a very small fraction of the resistance of the path $x_{1} x_{2}$, and that this ratio will not appreciably change in time since the negative susceptibility of the higher neurons is assumed to be very little greater than that of the lower neuron. We have thus changed an instinct into a habit in such a manner that the resistance of the paths leading from $S_{1}$ to $M_{1}$ is much less than it was under native conditions. In so far the case is like the one discussed above. But it is very different in this respect, that whenever now $S_{1}$ is stimulated, the nervous current takes the path exclusively over the higher center $S^{\prime} M^{\prime}$, whereas in the former case it would take the path exclusively over the lower center $x_{1} x_{2}$. The great significance of this difference for an animal's life is clear. In the former case, the reflex arch $S_{1} M_{2}$ has practically lost its connection, its functional communication with other reflex arches, $S_{2} M_{2}, S_{3} M_{3}$, etc. In the latter, the reflex arch has been brought into much closer connection with the other reflex arches, so that at any time it can enter into functional relations with them and those modifications of the response can be brought about which I have described in some detail in my previous article.

It is not, and cannot be, my task here to describe in detail what goes on in the nervous system in any definite situation occurring in a particular person's life. What I wished to show is 
merely this, that under the assumption of a very few principles of nervous function, pictured in mechanical terms, the most varied adaptations of the nervous system can easily be comprehended without any necessity for introducing mythological powers of which no one can tell us whence they come and whither they go. A mere change in the quantitative relations of the properties which the neurons are assumed to possess, leads to the result that a habit, instead of being less modifiable than the instinct of which it represents a strengthened form, is on the contrary vastly more modifiable than this instinct. If we only knew more about human and animal instincts and habits and the relations between them, we could derive from this experimental knowledge the quantitative relations which we had to assume quite arbitrarily; and we could understand quite well the functions of the nervous system even if the study of the anatomy and physiology of the nervous system were doomed to remain forever on the present level of development - a prophecy which, of course, no one will make.

Let us show in another case how we can explain a special form of habit if only the quantitative relations of our principles are properly selected. On page 304 of my previous paper I applied the theory to that form of habit which I called sensory condensation. The explanation there given is open to criticism because it does not take into account the quantitative aspect of the susceptibility of the neurons. I had to be very brief at that time. I shall now discuss the same case, going a little more into detail. We supposed that both $S_{1}$ and $S_{2}$ (see Fig. I above) were stimulated, but $S_{1}$ more strongly than $S_{2}$. Let us suppose that the resistances of all neurons are originally the same, and that the positive susceptibility of each of the neurons $x_{1} S^{\prime}, y_{1} S^{\prime}$, $S^{\prime} M^{\prime}, M^{\prime} x_{2}$ and $M^{\prime} y_{2}$ is three times that of either $x_{1} x_{2}$ or $y_{1} y_{2}$. (If we restrict ourselves to the establishing of the habit and neglect the forgetting, we need not make any assumption concerning the negative susceptibilities.) We found above that under these assumptions the stimulation of the single point $S_{1}$, however prolonged, would not change the relative resistances of the neurons $x_{1} S^{\prime}, S^{\prime} M^{\prime}, M^{\prime} x_{2}$ and $x_{1} x_{2}$. All these resistances, although absolutely diminished, would still be equal to each 
other. But when $S_{2}$ is stimulated simultaneously with, but more weakly than, $S_{1}$, the current in $S^{\prime} M^{\prime} x_{2}$ is increased by some flux coming from $S_{2}$. This reduces the resistance of the neuron $S^{\prime} M^{\prime}$, and also of $M^{\prime} x_{2}$, to less than the resistance of $x_{1} x_{2}$. In consequence of this reduction more of the flux coming from $S_{1}$ takes the path $x_{1} S^{\prime}$, less the path $x_{1} x_{2}$ than at first. The increased flux in $x_{1} S^{\prime}$ reduces the resistance of the neuron $x_{1} S^{\prime}$. It is clear that the longer all this goes on the more must the path $x_{1} S^{\prime} M^{\prime} x_{2}$ be favored with respect to resistance change in comparison with $x_{1} x_{2}$. Finally the existence of the path $x_{1} x_{2}$ may be entirely neglected, for its resistance, while it has steadily decreased, has become very much larger than the resistance of the longer path $x_{1} S^{\prime} M^{\prime} x_{2}$ whose resistance has so much more rapidly decreased.

Thus far, however, the establishment of a habit of the kind which we called sensory condensation has not been explained. If $S_{1}$ alone is now stimulated, no appreciable current would occur in $M^{\prime} y_{2}$, since the resistance of this neuron is by this time many times that of either of the paths leading from $S_{1}$ to $M_{1}$. If $S_{2}$ alone is stimulated the response would occur at $M_{1}$, not also at $M_{2}$. If $S_{2}$ is stimulated and $S_{1}$ at the same time, the response would again occur only at $M_{\mathrm{l}}$. It is necessary, in order to bring about this special form of habit, sensory conden sation, that double stimulations of both kinds, $\mathrm{S}_{1}$ strong with $S_{2}$ weak, and $S_{2}$ strong with $S_{1}$ weak, occur in frequent alternation from the start. Suppose the resistance of the path $x_{1} S^{\prime} M^{\prime} x_{2}$ has been reduced only to very little less than three times the resistance of $x_{1} x_{2}$, and now $S_{2}$ is stimulated strongly and $S_{2}$ weakly. Then the flux from $S_{2}$ will not take its way towards $M_{1}$, but most of it passes over $y_{1} y_{2}$ towards $M_{2}$ and most of the flux from $S_{1}$ takes its way over $S^{\prime} M^{\prime}$ towards $M_{2}$. Thus the resistance of $y_{1} S^{\prime} M^{\prime} y_{2}$ can by steps decrease to much less than $y_{1} y_{2}$. I say by steps, because this occurs while the symmetrically corresponding change on the left side of the figure, discussed above, has its pauses. The total outcome then is, that the existence of both the paths $x_{1} x_{2}$ and $y_{1} y_{2}$ may be disregarded, because the resistance of the paths $x_{1} S^{\prime} M^{\prime} x_{2}$ and $y_{1} S^{\prime} M^{\prime} y_{2}$ has become incomparably smaller than the resistance of either $x_{1} x$ - 
or $y_{1} y_{2}$, although absolutely these latter resistances have decreased too. Whenever now a single stimulus is applied to either $S_{1}$ or $S_{2}$, the flux takes its path over $S^{\prime} M^{\prime}$ and dividing at $M^{\prime}$ produces a double response, at $M_{1}$ and $M_{2}$. This is what we called sensory condensation.

It may be well to illustrate this kind of habit. A child learning to play the piano has before him two notes to which he is expected to respond by simultaneous movement of two fingers. One note is seen by foveal vision and the corresponding finger movement occurs with definiteness. The other note is seen by extra-foveal vision and the corresponding movement lacks definiteness. The child tries again and looks now at the note whose movement was not properly executed. Everything is reversed. The finger movement which was previously done badly is now done well, and the movement which was previously done well, is done badly. After many trials, on the whole alternating as to the favorable note and finger, the child becomes able to perform both movements simultaneously with definiteness although he is looking at only one of the notes - no matter which one he is looking at.

Summarizing, then, we may say that there is no justification for the remark not infrequently heard, that psychology can make no further theoretical progress, that we have to wait until the anatomy and physiology of the nervous system have made a long step in advance. We need not be discouraged. Experimental knowledge of habits and of their development out of instincts will enable us to obtain a scientific theory of human and animal activity by permitting us to determine the quantitative values which for the present we have to assume quite arbitrarily.

(I may use this opportunity for correcting a mistake in the description of the establishment of the 'variation of response' in my previous article, p. 303 . In the fifth line from the bottom, between the words actually and than, the word ' greater' ought to be substituted for ( less.') 1

( $T o$ be concluded.)

1 The MS. of this article was received September I, I908. - ED. 